\section{Impacto del trabajo a turnos \\ sobre la salud y la satisfacción laboral de los trabajadores en España}

\author{
María-Carmen Sánchez-Sellero (https://orcid.org/0000-0003-3730-8415)' \\ Departamento de Economía, Facultad de Economía y Empresa, \\ Universidade da Coruña, San Vicenzo de Elviña, España.
}

Resumen: Este artículo analiza la satisfacción y la salud en los trabajadores a turnos, así como las posibles relaciones entre esos términos a partir de los datos españoles de la 6a Encuesta Europea de Condiciones de Trabajo de 2015, publicada en 2017. Utilizamos matriz de correlaciones, análisis de correspondencias y regresión logística. Los trabajadores a turnos rotativos presentaron porcentajes menores de estar satisfechos o muy satisfechos con su trabajo, siendo el efecto negativo más acusado en la satisfacción que en la salud. Se evidencia para todos los turnos de trabajo que los que gozan de "muy buena salud" son los que se encuentran "muy satisfechos" laboralmente. La satisfacción de los trabajadores a turnos es 1,047 veces menor que los que no están a turnos, mientras que los que gozan de muy buena salud están 6,325 veces más satisfechos que su categoría de referencia. Consideramos necesario un estudio longitudinal pues la permanencia en el trabajo a turnos puede incrementar los efectos negativos en el trabajador.

Palabras clave: Turnos de trabajo. Salud. Satisfacción laboral. Estudio de correlaciones.

\section{Impact of shift work on health and job satisfaction of workers in Spain}

Abstract: This article analyzes the satisfaction and health of shift workers, as well as the possible relationships between these terms based on the Spanish data of the 6th European Survey of Working Conditions of 2015, published in 2017. We use matrix of correlations, analysis of correspondences and logistic regression. Rotating shift workers had lower percentages of being satisfied or very satisfied with their work, with the negative effect more pronounced in satisfaction than in health. It is evident for all work shifts that those who enjoy "very good health" are those who are "very satisfied" at work. The satisfaction of shift workers is 1,047 times lower than those who are not in shift, while those who enjoy very good health are 6,325 times more satisfied than their reference category. We consider necessary a longitudinal study because the permanence in shift work could increase the negative effects on the worker.

Keywords: Shift work. Health. Job satisfaction. correlation studies.
Recebido: 26.11 .19

Aprovado: 30.09 .20

I. María-Carmen

Sánchez-Sellero

es doctora en

ciencias económicas

y empresariales,

profesora titular en

el Departamento

de Economía,

Facultad de

Economía y Empresa,

Universidade da

Coruña, Campus de

Elviña, España.

<c.sanchez@udc.es>.

1. El Estatuto de los Trabajadores en España dice lo siguiente: "Se considera trabajo a turnos toda forma de organización del trabajo en equipo según la cual los trabajadores ocupan sucesivamente los mismos puestos de trabajo, según un cierto ritmo, continuo o discontinuo, implicando para el trabajador la necesidad de prestar sus servicios en horas diferentes en un periodo determinado de días o de semanas" (artículo 36.3. del Boletín Oficial del Estado, de 24 de octubre de 2015). 


\section{Introducción}

D e todos es conocido que el trabajo a turnos ${ }^{1}$ no deja indiferente al trabajador que lo realiza, ni al mercado laboral al que pertenece. Si no fuera así, no existirían las "compensaciones" en forma de dinero y/o de días libres que se conceden a los trabajadores en turnos rotativos después de trabajar en un turno nocturno. Este estudio tiene su punto de partida en el trabajo a turnos, y más concretamente su impacto sobre la salud y sobre la satisfacción de los trabajadores.

Se pueden considerar las diferentes formas de entender este tipo de trabajo dependiendo de los países en los que se analice. En Brasil, la jornada de trabajo está regulada por el artículo 70, XIII de la Constituição Federal y por el artículo 58 de la Consolidação das Leis do Trabalho; de ahí empleados y empleadores encuentran las normas legales que deben cumplirse en el caso de tipos de horario de trabajo. Lo más común es que los trabajadores realicen sus funciones durante 8 horas diarias y 44 horas semanales. Sin embargo, existe la posibilidad de compensación y turnos de relevo, en cuyo caso la actividad se organiza en escalas, pudiendo ser $5 \times 1,5 \times 2$, $4 \times 2,6 \times 1,12 \times 36,18 \times 36,24 \times 48$. Por ejemplo, en la escala $4 \times 2$ el empleado trabaja durante 4 días consecutivos, en turnos de 11 horas y tiene 2 días libres. En España, el informe de la sexta EWCS-España (2015) muestra la siguiente distribución de los trabajos a turnos: aquellos que trabajan en turnos fijos de mañana, tarde o noche; los que trabajan a turnos rotativos o alternativos; y los que trabajan a turno partido (con un descanso de al menos 4 horas entremedias).

El interés de este tema reside en varias razones: las condiciones de trabajo y de empleo son determinantes fundamentales de la salud y de bienestar, siendo el lugar de trabajo un entorno idóneo para llevar a cabo intervenciones con un grado directo de participación (García et alii, 2016); en salud laboral, es posible afirmar que el trabajo no es neutral respecto a la salud (Rodriguez, 2013). Las investigaciones en los últimos años muestran la presencia de muchas repercusiones negativas sobre la salud de los trabajadores a turnos. Desde una perspectiva psicológica destacan los trastornos del sueño, síndrome de fatiga crónica, estrés laboral, sintomatología depresiva, así como problemas familiares y sociales (Fernández \& Piñol, 2000). A los trabajos a turnos también se les atribuye un mayor número de problemas laborales (menor rendimiento, absentismo y accidentes de trabajo), en comparación con los trabajadores con un horario convencional (Fernández \& Piñol, 2000).

En la literatura existente se trata o bien el efecto del trabajo a turnos sobre la salud, o bien el efecto sobre la satisfacción laboral, pero son más escasos los estudios que abordan el efecto del trabajo a turnos sobre la salud y la satisfacción laboral, 
buscando posibles relaciones entre estas variables. En parecidos términos aunque no iguales, Bernstorff (2008) tuvo como objetivo, correlacionar la satisfacción laboral, la evaluación del desempeño por competencia individual, el examen de la salud ocupacional y el absentismo laboral. Además, la mayoría de las investigaciones se suelen referir a colectivos de trabajadores muy concretos pertenecientes a actividades específicas. No encontramos ninguna investigación que analice estas relaciones concretas (trabajo a turnos, salud y satisfacción) a partir de una base de datos pública y representativa de todos los trabajadores de un país; ésta es la mayor contribución de este trabajo. Este hecho hace que los resultados y conclusiones sean generalizables a un ámbito mayor que si se tratase por ejemplo de una muestra obtenida de una empresa u organización.

Sentirse satisfecho y sentirse sano son dos percepciones subjetivas, ya que dos personas pueden declarar distintos niveles de satisfacción y salud estando en idénticas situaciones. Queremos vincular el trabajo a turnos con ambas percepciones. También es de nuestro interés conocer el efecto del paso del tiempo en las relaciones anteriores. Por todo ello, los objetivos propuestos intentan dar contestación a las siguientes cuestiones o hipótesis de trabajo:

Hipótesis 1: Influencia del trabajo a turnos sobre la salud.

Hipótesis 2: Influencia del trabajo a turnos sobre la satisfacción laboral.

Hipótesis 3: Establecimiento de correlaciones entre las variables trabajo a turnos, salud y satisfacción.

Hipótesis 4: Efecto del tiempo en las relaciones entre las variables trabajo a turnos, salud y satisfacción laboral.

El proceso o pasos de esta investigación se pueden resumir en la Figura 1.

FIGURA 1

PASOS A DESARROLLAR EN EL PROCESO DE INVESTIGACIÓN

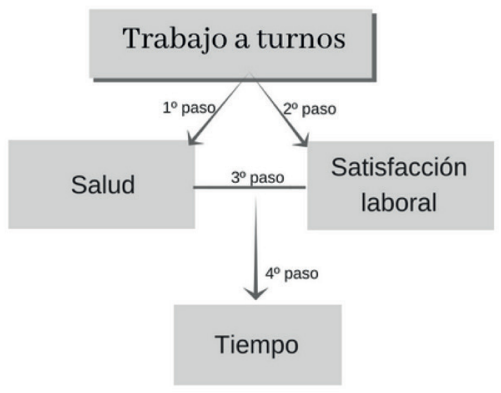


Tras esta introducción, planteamos un epígrafe de marco teórico, uno de material y métodos empleados, uno de resultados y otro de discusión, para acabar con las conclusiones del trabajo.

\section{Marco teórico}

Los trabajos a turnos, en horarios irregulares, en días de descanso, la extensión de horas diarias de trabajo etc. son temas importantes desde la perspectiva de salud laboral (Parra, 2003). Parra afirma que los turnos de noche o una jornada laboral extensa pueden actuar como agravantes de un problema de salud previo existente. De ello se podría derivar que las consecuencias negativas sobre la salud presentan especial incidencia en los trabajadores que realizan turno nocturno. En el trabajo nocturno se altera la fisiología normal del organismo; no existe una adaptación ni siquiera en los trabajadores nocturnos permanentes, porque no hay una transformación en seres humanos nocturnos. Entre sus recomendaciones y para prevenir los problemas del trabajo nocturno, Parra (2003) recomienda:

- Buscar alternativas del trabajo a turnos.

- Reducir el número de turnos de noche consecutivos.

- Prohibir los cambios de turno en poco tiempo, existiendo una pausa mínima de 12 horas entre la salida de un turno y la entrada a otro.

- Otorgar el máximo número de fines de semana libres.

- Organizar pocos turnos largos y de sobretiempo.

- No usar turnos largos para tareas pesadas.

- Vigilar las horas de entrada y salida a turnos.

- Diseñar sistemas para que sean lo más regulares posibles.

- Propiciar un número mayor de descansos en el turno de noche.

- Proveer estancias adecuadas para dormir, cuando se trabaja fuera de la residencia habitual.

De igual modo, Gil-Monte (2012) señala que según sea la organización del tiempo de trabajo, ya sea la duración y tipo de jornada, pausas de trabajo, trabajo en festivos, trabajo a turnos y nocturno etc. pueden representar factores de riesgo para la salud. La investigación de Iskra-Golec et alii (2017) documenta que las consecuencias del trabajo a turnos pueden depender de los parámetros del sistema de turnos; 
descubrieron que los sistemas de rotación rápida (1×3 turnos del mismo tipo en fila) y el trabajo diurno son menos disruptivos biológicamente que los sistemas de rotación más lentos y el trabajo de tarde y noche.

Existen trastornos del ritmo circadiano de origen extrínseco, como los originados por el trabajo a turnos o los secundarios a unos horarios vigilia-sueño inapropiados (Peraita-Adrados, 2005). En esta alteración del ritmo circadiano, los síntomas de insomnio o hipersomnia son propios de jornadas o turnos laborales que se solapan con el periodo normal del sueño, permaneciendo el trabajador alerta en un momento inadecuado de su ciclo sueño-vigilia. Las condiciones de trabajo tanto de los trabajadores que hacen turnos nocturnos o de madrugada, como de aquellos con rotación de los turnos (estos últimos necesitan un tiempo para adaptar su ciclo sueño-vigilia a la nueva situación) conllevan una reducción de las horas de sueño con un desajuste del ritmo circadiano de sueño-vigilia (Gállego et alii, 2007).

En cuanto al concepto de satisfacción laboral, existe una gran cantidad de estudios sobre el tema, entendiendo como tal un constructo multidimensional (Porcel-Gálvez et alii, 2014), ya que en la explicación de este concepto influyen múltiples factores o variables (Sánchez-Sellero et alii, 2014; Boria-Reverter et alii, 2012), aunque la mayoría de las investigaciones reflejan que son las variables inherentes al trabajo las que ejercen mayor influencia (Dueñas et alii, 2010; Robles-García et alii, 2005). En palabras de Iglesias et alii (2011), la satisfacción laboral es una cuestión que explica algunos de los más importantes resultados del mercado laboral. Conviene tener en cuenta, como bien dicen Santos et alii (2012), que los objetivos organizacionales no se logran sin un fuerte compromiso afectivo (Hitka et alii, 2015; Lorincová et alii, 2019) y satisfacción por parte de los trabajadores en los resultados de la organización.

En la revisión de la literatura hecha por Dall'Ora et alii (2016) sobre características del trabajo a turnos y su impacto en el rendimiento, pudieron concluir que los turnos rotativos se asociaron con peores resultados de desempeño laboral, mientras que los turnos nocturnos fijos parecían permitir la resincronización. Sin embargo, la satisfacción laboral de los empleados que trabajan noches fijas se redujo. Los descansos oportunos tuvieron un impacto positivo en la fatiga y el estado de alerta de los empleados, mientras que los retornos rápidos entre los turnos parecían aumentar la fatiga. El efecto de las características del trabajo a turnos sobre los resultados en los estudios revisados es consistente en todos los sectores ocupacionales.

Khammar et alii (2017) parten de la premisa que el trabajo a turnos influye negativamente en la calidad de la vida laboral de los trabajadores. En su estudio empírico 
concreto, las personas que habían elegido voluntariamente el trabajo a turnos tenían mayor satisfacción laboral que las que lo hacían forzadas a trabajar a turnos. Los resultados de Ferri et alii (2016) sugieren que las personas con horario nocturno rotativo presentaron promedios más bajos en los ítems de satisfacción laboral, calidad y cantidad de sueño, síntomas de fatiga crónica, psicológicos y cardiovasculares más frecuentes en comparación con los trabajadores de turno diurno, de manera estadísticamente significativa. En la investigación de Šimunić y Gregov (2012), el componente evaluativo de satisfacción laboral fue más alto en las personas de turno matutino; además, consideran que el tipo de rotación del turno es importante.

\section{Material y métodos}

La base de datos es la sexta EWCS-España. Se encuadra en la "6a Encuesta Europea de Condiciones de Trabajo" (European Working Conditions Survey). Se publicó en 2017, corresponde a la edición de 2015 de la Encuesta Nacional de Condiciones de Trabajo. Esta es una encuesta multinacional (35 países) que la Fundación Europea para la Mejora de las Condiciones de Vida y de Trabajo (Eurofound) realiza cada cinco años. En 2015 se entrevistó a 43.850 trabajadores, 3.364 de ellos en España, sobre diferentes aspectos de su vida laboral, como el horario de trabajo, el lugar de trabajo, la exposición a riesgos físicos y psicosociales, la salud y el bienestar, entre otros. Los requisitos para formar parte de la encuesta es tener 15 años o más (en el caso de algunos países como España, fue de 16 o más años, ya que ésta es la edad mínima legal para trabajar), ser residente en los países incluidos en la encuesta, y estar en 'situación de empleo' (personas que realizaron al menos una hora de trabajo a cambio de una remuneración en la semana anterior a la entrevista).

En España, la muestra se estratificó por Comunidades Autónomas y grado de urbanización (5 categorías). Inicialmente, en cada estrato se seleccionaron proporcionalmente al número de personas empleadas en cada estrato, 825 unidades primarias de muestreo (UPM) sobre la base de secciones censales. Las estadísticas de población se obtuvieron de la Encuesta de Población Activa (EPA) correspondientes al segundo trimestre del año 2014. En cada sección censal se dispuso de un registro de calles y números de cada calle, por lo que se realizó una selección aleatoria de direcciones dentro de cada unidad primaria de muestreo (Primary Sampling Unit PSU). Por último, dentro de cada unidad familiar se seleccionó aleatoriamente a la persona con trabajo que estuvo de cumpleaños más recientemente.

De la sexta EWCS-España seleccionamos inicialmente las variables Turnos, Tipos de Turnos, Salud y Satisfacción. En todas ellas eliminamos los códigos que se refieren a "No sabe", "No contesta" para que los cálculos estadísticos no alteren los resul- 
tados y se refieran solo a las contestaciones válidas. La variable Tipos de Turnos procede si en la pregunta Turnos=1 (contestaron Si). Se enuncian así:

Usted trabaja... Por turnos: 1. Si, 2. No.

Usted trabaja... 1. A turno partido (con un descanso de al menos 4 horas entremedias), 2. A turnos fijos (mañana, tarde o noche), 3. A turnos rotativos/alternativos, 4 . Otro.

¿Cómo es su salud en general? Diría que es...1. Muy buena, 2. Buena, 3. Aceptable, 4. Mala, 5. Muy mala.

En general, ¿̇está muy satisfecho, satisfecho, no muy satisfecho o nada satisfecho con las condiciones de trabajo de su principal trabajo remunerado? Diría que está... 1. Muy satisfecho, 2. Satisfecho, 3. No muy satisfecho, 4. Nada satisfecho.

En la regresión logística utilizamos otras variables relativas a características personales del trabajador (género, edad), y características propias del trabajo (sueldo, posibilidades de ascenso, reconocimiento del trabajo, compañeros, motivación organización, tipo de contrato, sector de actividad, tipo de empleado según empleador), además de las variables trabajar a turnos, salud y satisfacción.

Empleamos tablas de contingencias, matriz de correlaciones, análisis de correspondencias y regresión logística binaria, todo ello con el programa SPSS 24. Mediante el análisis de correspondencias estudiamos desde un punto de vista gráfico las relaciones de dependencia de variables cualitativas a partir de una tabla de contingencia. Para ello asociamos a cada categoría un punto en el espacio $\mathrm{R}^{n}$ (generalmente un plano, $\mathrm{n}=2$ ) de forma que la cercanía/lejanía entre los puntos reflejan relaciones de proximidad entre las categorías. Aplicamos el método de normalización simétrica, según el cual, para cada dimensión (o eje), las puntuaciones de fila son la media ponderada de las puntuaciones de columna divididas por el valor propio coincidente, y para las puntuaciones de columna al revés. En el origen de coordenadas se encuentran las categorías similares a la media de las filas (o columnas). En términos generales, se interpreta que dos categorías próximas en el gráfico indican asociación entre ellas, mucho más cuando se encuentran lejos del centro de gravedad (origen de coordenadas).

Mediante la regresión logística (Logit) queremos modelizar cómo influye en la probabilidad de aparición de un suceso, en este caso dicotómico al ser una regresión logística binaria, un conjunto de variables explicativas (ya sean dicotómicas, cate- 
góricas y/o cuantitativas). Sea $Y$ una variable dependiente binaria (con dos posibles valores: 0 y 1$)$, y un conjunto de $k$ variables independientes $\left(X_{1}, X_{2}, \ldots, X_{k}\right)$, observadas que sirven para predecir/explicar el valor de $Y$. El objetivo consiste en determinar:

$P\left(Y=1 / X_{1}, X_{2}, \ldots, X_{k}\right)$

$P\left(Y=0 / X_{1}, X_{2}, \ldots, X_{k}\right)=1-\left(Y=1 / X_{1}, X_{2}, \ldots, X_{k}\right)$

El modelo de regresión logística binaria establece la siguiente relación entre la probabilidad de que ocurra el suceso, dado que en cada caso se presentan los valores

$\left(X_{1}=x_{1}, X_{2}=x_{2}, \ldots, X_{\mathrm{k}}=x_{\mathrm{k}}\right)$ :

$P\left(Y=1 / x_{1}, x_{2}, \ldots, x_{k}\right)=\frac{1}{1+e^{\left(-\beta_{0}-\beta_{1} x_{1}-\beta_{2} x_{2}-\ldots-\beta_{k} x_{k}\right)}}$

Se trata de hallar los coeficientes $\left(\beta_{0}, \beta_{1}, \beta_{2}, \ldots, \beta_{k}\right)$ que mejor se ajusten a la expresión funcional.

Se conoce como odds-ratio al cociente de probabilidades:

odds-ratio $=\frac{P\left(Y=1 / x_{1}, x_{2}, \ldots, x_{k}\right)}{1-P\left(Y=1 / x_{1}, x_{2}, \ldots, x_{k}\right)}=e^{\beta_{0}+\beta_{1} x_{1}+\beta_{2} x_{2}+\ldots+\beta_{k} x_{k}}$

Tomando logaritmos neperianos en la expresión anterior, nos queda la expresión lineal:

$\operatorname{Ln}\left[\frac{P\left(Y=1 / x_{1}, x_{2}, \ldots, x_{k}\right)}{1-P\left(Y=1 / x_{1}, x_{2}, \ldots, x_{k}\right)}\right]=\beta_{0}+\beta_{1} x_{1}+\beta_{2} x_{2}+\ldots+\beta_{k} x_{k}$

Un odds-ratio próximo a 1 , es decir, un coeficiente $\beta_{i}$ cercano a cero, indicará que cambios en esa variable explicativa no tendrá efecto alguno sobre la variable dependiente. La estimación en modelos Logit se realiza mediante el método de Máxima Verosimilitud. Además, en este tipo de modelos no resulta posible interpretar directamente las estimaciones de los parámetros $\beta$, ya que son modelos no lineales. Por ello, vamos a fijarnos en el signo de los estimadores. Si el estimador es positivo, significará que incrementos en la variable asociada causan incrementos en $P(Y=1)$. Por el contrario, si el estimador tiene signo negativo, supondrá que incrementos en la variable asociada causarán disminuciones en $P(Y=1)$. Conviene observar que los p-valores (Sig.) inferiores a 0,05, para un nivel de significación $\alpha=0,05$, conlleva rechazar la hipótesis nula de que dichos coeficientes son nulos; y por tanto, las variables asociadas a los mismos son relevantes en el modelo. 


\section{Resultados}

Hipótesis 1: Influencia del trabajo a turnos sobre la salud.

El 23\% de los encuestados reconoce trabajar a turnos; de éstos un 49,8\% declaran trabajar a turnos fijos mientras que un 40,9\% a turnos rotativos/alternativos (véase distribuciones marginales de las variables, Tabla 1). El 78,5\% del total de encuestados cree que su salud es buena o muy buena (Tabla 1), aunque en los que trabajan a turnos rotativos es del $77,3 \%$, es decir, el porcentaje varía -1,2\% respecto al total (Tabla 2). La tabla 2 se refiere únicamente a los trabajadores que trabajan a turnos; en ella, $N=766$. En ambas tablas se ha ponderado por $w 4$, siguiendo las indicaciones de la metodología de la encuesta.

Hipótesis 2: Influencia del trabajo a turnos sobre la satisfacción laboral.

En cuanto a la satisfacción, el $81,7 \%$ del total de los trabajadores afirmaron estar satisfechos o muy satisfechos con su trabajo (Tabla 1 ), disminuyendo en $0,7 \%$ ese porcentaje en el total de los que trabajan a turnos. Sin embargo, los trabajadores a turnos rotativos están satisfechos o muy satisfechos con su trabajo únicamente el $77 \%$, sufriendo una variación de-4,7\% respecto al total (véase Tabla 2).

Hipótesis 3: Establecimiento de correlaciones entre las variables trabajo a turnos, salud y satisfacción.

Para conocer las asociaciones entre estas variables se utiliza una matriz de correlaciones (Tabla 3), donde también se señala cuáles de estas relaciones son significativas ( $p$-valor menor que 0,05 ).

Como la correlación más alta es la existente entre la salud y la satisfacción laboral $(0,226)$, procede realizar un análisis de correspondencias entre estas dos variables. En la Tabla 4 se visualiza el resumen del análisis de correspondencias y el examen de los puntos de fila y columna entre las variables salud y satisfacción laboral, mientras que la Figura 2 corresponde a su representación gráfica.

Con el fin de encontrar dependencias entre estas variables, en la Tabla 5 hacemos también una regresión logística binaria donde la variable dependiente es la satisfacción laboral recodificada en dicotómica: (1-satisfecho-muy satisfecho, 0-nada satisfecho-no muy satisfecho) y siendo las variables independientes, variables de tipo personal del trabajador y otras variables propias del trabajo, además de las variables trabajar a turnos y salud. 
TABLA 1

DISTRIBUCIONES MARGINALES DE LAS VARIABLES TURNOS,

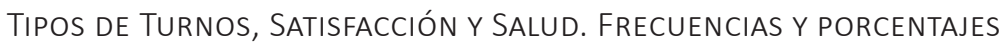

\begin{tabular}{|c|c|c|c|c|c|}
\hline & & Frecuencia & Porcentaje & Porcentaje válido & $\begin{array}{l}\text { Porcentaje } \\
\text { acumulado }\end{array}$ \\
\hline \multirow{5}{*}{ 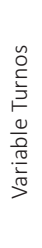 } & Sí & 772 & 23,0 & 23,0 & 23,0 \\
\hline & No & 2583 & 76,8 & 77,0 & 100,0 \\
\hline & Total & 3355 & 99,7 & 100,0 & \\
\hline & $\begin{array}{l}\text { Perdidos (No } \\
\text { sabe, NC) }\end{array}$ & 9 & 0,3 & & \\
\hline & Total & 3364 & 100,0 & & \\
\hline \multirow{7}{*}{ 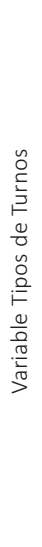 } & $\begin{array}{l}\text { A turno partido } \\
\text { [con un descanso } \\
\text { de al menos } 4 \text { ho- } \\
\text { ras entremedias] }\end{array}$ & 63 & 1,9 & 8,3 & 8,3 \\
\hline & $\begin{array}{l}\text { A turnos fijos } \\
\text { [mañana, tarde o } \\
\text { noche] }\end{array}$ & 382 & 11,4 & 49,8 & 58,0 \\
\hline & $\begin{array}{l}\text { A turnos rotati- } \\
\text { vos-alternativos }\end{array}$ & 314 & 9,3 & 40,9 & 98,9 \\
\hline & $\begin{array}{l}\text { Otro [espontá- } \\
\text { neo] }\end{array}$ & 8 &, 2 & 1,1 & 100,0 \\
\hline & Total & 767 & 22,8 & 100,0 & \\
\hline & $\begin{array}{l}\text { Perdidos (No } \\
\text { sabe, NC) }\end{array}$ & 5 & 0,1 & & \\
\hline & Total & 772 & & & \\
\hline \multirow{7}{*}{ 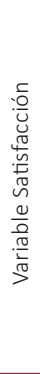 } & Muy satisfecho & 807 & 24,0 & 24,0 & 24,0 \\
\hline & Satisfecho & 1937 & 57,6 & 57,7 & 81,7 \\
\hline & $\begin{array}{l}\text { No muy satis- } \\
\text { fecho }\end{array}$ & 474 & 14,1 & 14,1 & 95,8 \\
\hline & Nada satisfecho & 142 & 4,2 & 4,2 & 100,0 \\
\hline & Total & 3359 & 99,9 & 100,0 & \\
\hline & $\begin{array}{l}\text { Perdidos (No } \\
\text { sabe, NC) }\end{array}$ & 5 & 0,1 & & \\
\hline & Total & 3364 & 100,0 & & \\
\hline \multirow{8}{*}{ 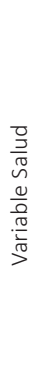 } & Muy buena & 780 & 23,2 & 23,2 & 23,2 \\
\hline & Buena & 1858 & 55,2 & 55,2 & 78,5 \\
\hline & Aceptable & 632 & 18,8 & 18,8 & 97,2 \\
\hline & Mala & 83 & 2,5 & 2,5 & 99,7 \\
\hline & Muy mala & 9 & 0,3 & 0,3 & 100,0 \\
\hline & Total & 3362 & 100,0 & 100,0 & \\
\hline & $\begin{array}{l}\text { Perdidos (No } \\
\text { sabe, NC) }\end{array}$ & 2 & 0,0 & & \\
\hline & Total & 3364 & 100,0 & & \\
\hline
\end{tabular}

Fuente: Elaboración propia a partir de la 6a Encuesta Europea de Condiciones de Trabajo-España 2015. 
TABLA 2

TABLA DE CONTINGENCIA (VALORES Y PORCENTAJES).

VARIABLES: TURNOS, SALUd Y SATISFACCIÓN CON EL TRABAJO

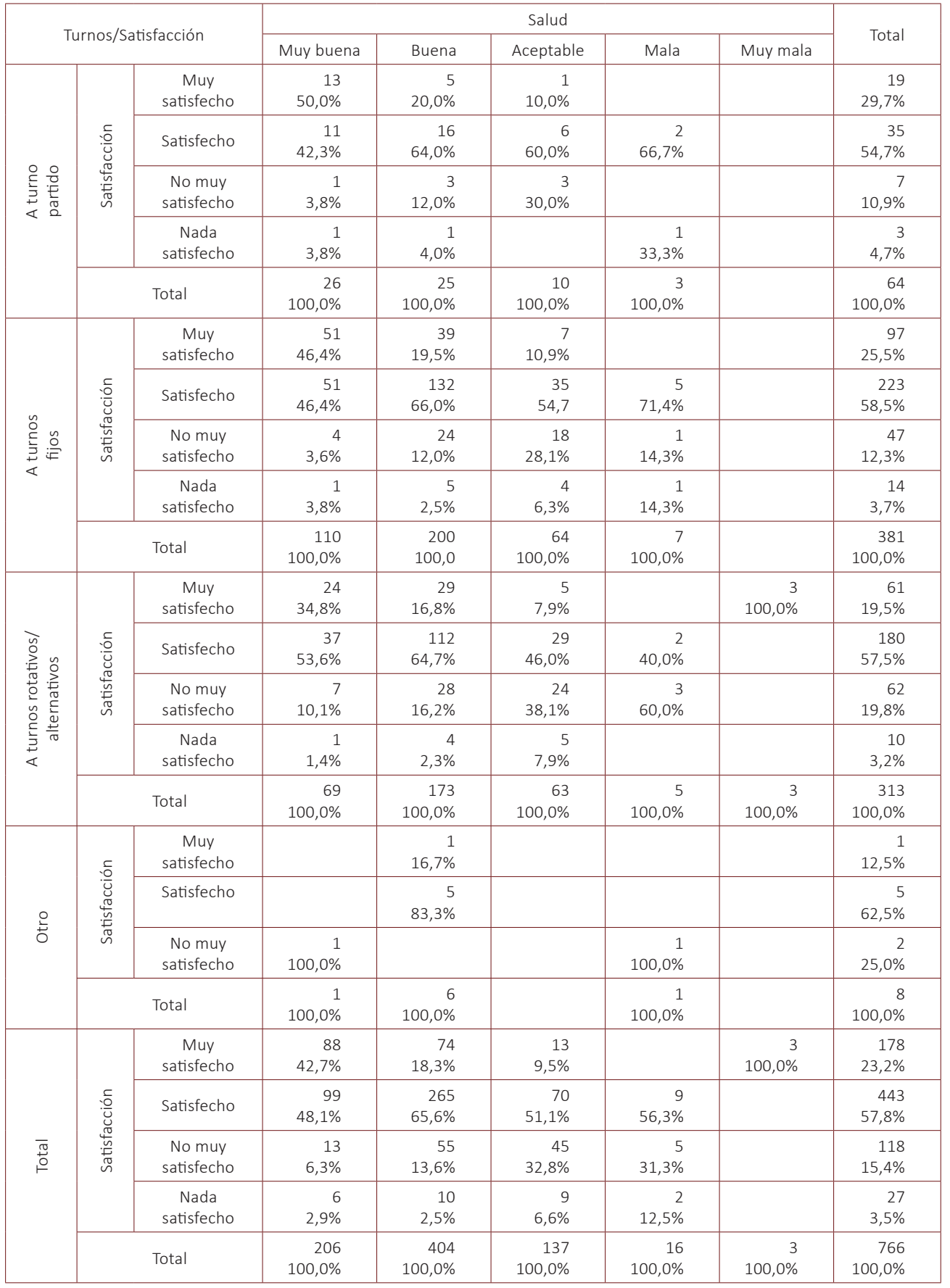

Fuente: Elaboración propia a partir de la 6ạ Encuesta Europea de Condiciones de Trabajo-España 2015. 
TABLA 3

MATRIZ DE CORRELACIONES. VARIABLES:

TURNOS, SALUD Y SATISFACCIÓN CON EL TRABAJO

\begin{tabular}{|c|c|c|c|c|}
\hline & & Salud & Satisfacción & Turnos \\
\hline \multirow{3}{*}{ Salud } & Correlación de Pearson & 1 & $0,226 * *$ & $0,090 *$ \\
\hline & Sig. (bilateral) & & 0,000 & 0,012 \\
\hline & $N$ & 3362 & 3359 & 766 \\
\hline \multirow{3}{*}{ Satisfacción } & Correlación de Pearson & $0,226 * *$ & 1 & $0,096 * *$ \\
\hline & Sig. (bilateral) & 0,000 & & 0,008 \\
\hline & $\mathrm{N}$ & 3359 & 3359 & 766 \\
\hline \multirow{3}{*}{ Turnos } & Correlación de Pearson & $0,090 *$ & $0,096 * *$ & 1 \\
\hline & Sig. (bilateral) & 0,012 & 0,008 & \\
\hline & $\mathrm{N}$ & 766 & 766 & 766 \\
\hline
\end{tabular}

**. La correlación es significativa en el nivel 0,01 (bilateral).

*. La correlación es significativa en el nivel 0,05 (bilateral).

Fuente: Elaboración propia a partir de la 6ạ Encuesta Europea de Condiciones de Trabajo-España 2015.

FIGURA 2

ANÁLISIS DE CORRESPONDENCIAS.

MÉTOdO DE NORMALIZACIÓN SIMÉTRICA.

VARIABLES: SALUD Y SATISFACCIÓN CON EL TRABAJO

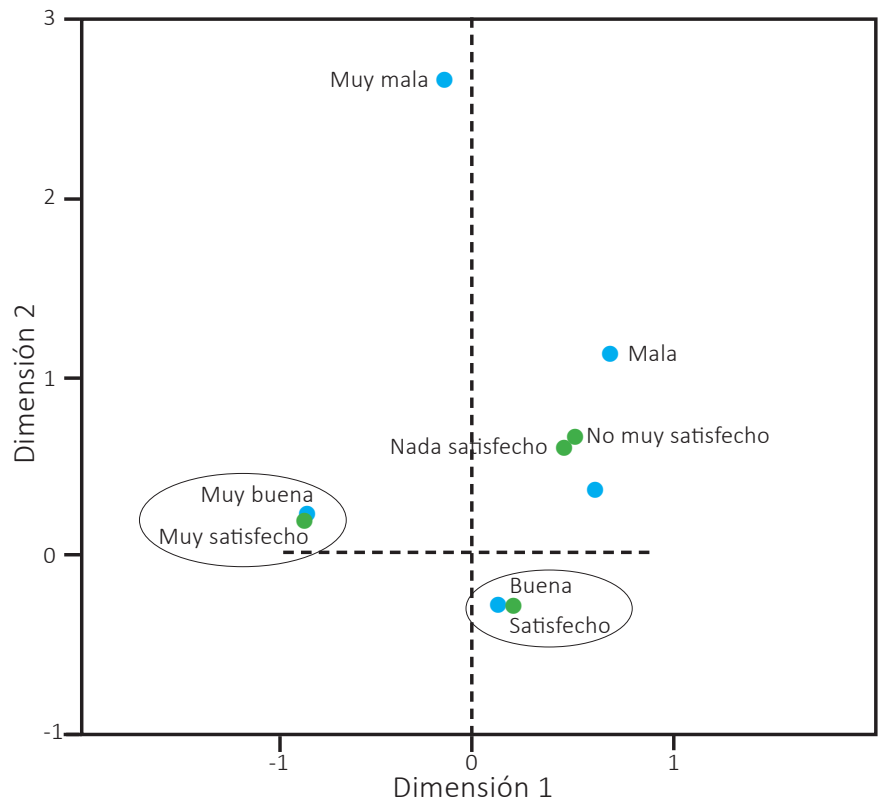

Q Q75- ¿Como es su salud en general? Diría que es...

Q Q88-En general, ¿está muy satisfecho, satisfecho, no muy satisfecho, o nada satisfecho con las

condiciones de trabajo de su principal trabajo remunerado? Diría que está...

Fuente: Elaboración propia a partir de la 6ạ Encuesta Europea de Condiciones de Trabajo-España 2015 
TABLA 4

ANÁLISIS DE CORRESPONDENCIAS.

CONTRIBUCIONES A LA INERCIA TOTAL DE CADA DIMENSIÓN, PUNTOS DE FILA Y PUNTOS DE COLUMNA.

VARIABLES: SALUD Y SATISFACCIÓN CON EL TRABAJO

\begin{tabular}{|c|c|c|c|c|c|c|c|c|c|}
\hline \multicolumn{9}{|c|}{ Resumen } & \\
\hline \multirow{3}{*}{ Dimensión } & \multirow{3}{*}{$\begin{array}{l}\text { Valor } \\
\text { propio }\end{array}$} & \multirow{3}{*}{ Inercia } & \multirow{3}{*}{$\begin{array}{l}\text { Chi- } \\
\text { cuadrado }\end{array}$} & \multirow{3}{*}{ Sig. } & \multicolumn{2}{|c|}{ Proporción de inercia } & \multicolumn{2}{|c|}{$\begin{array}{c}\text { Confianza para el valor } \\
\text { propio }\end{array}$} & \\
\hline & & & & & \multirow{2}{*}{ Explicada } & \multirow{2}{*}{ Acumulada } & \multirow{2}{*}{$\begin{array}{l}\text { Desviación } \\
\text { estándar }\end{array}$} & \multirow{2}{*}{$\frac{\text { Correlación }}{2}$} & \\
\hline & & & & & & & & & \\
\hline 1 & 0,257 & 0,066 & & & 0,785 & 0,785 & 0,018 & 0,130 & \\
\hline 2 & 0,134 & 0,018 & & & 0,212 & 0,998 & 0,018 & & \\
\hline 3 & 0,014 & 0,000 & & & 0,002 & 1,000 & & & \\
\hline Total & & 0,084 & 281,918 & $0,000^{a}$ & 1,000 & 1,000 & & & \\
\hline \multicolumn{10}{|c|}{ Puntos de filab } \\
\hline \multirow{3}{*}{ Satisfacción } & \multirow{3}{*}{ Masa } & \multicolumn{2}{|c|}{$\begin{array}{l}\text { Puntuación en } \\
\text { dimensión }\end{array}$} & \multirow{3}{*}{ Inercia } & \multicolumn{5}{|c|}{ Contribución } \\
\hline & & 1 & 2 & & \multicolumn{2}{|c|}{$\begin{array}{l}\text { Del punto en la inercia de } \\
\text { dimensión }\end{array}$} & \multicolumn{3}{|c|}{ De la dimensión en la inercia del punto } \\
\hline & & & & & 1 & 2 & 1 & 2 & Total \\
\hline $\begin{array}{l}\text { Muy } \\
\text { satisfecho }\end{array}$ & 0,240 & $-0,870$ & 0,170 & 0,048 & 0,708 & 0,052 & 0,981 & 0,019 & 1,000 \\
\hline Satisfecho & 0,577 & 0,189 & $-0,282$ & 0,011 & 0,081 & 0,343 & 0,465 & 0,535 & 1,000 \\
\hline $\begin{array}{l}\text { No muy } \\
\text { satisfecho }\end{array}$ & 0,141 & 0,561 & 0,675 & 0,020 & 0,173 & 0,481 & 0,570 & 0,428 & 0,998 \\
\hline $\begin{array}{l}\text { Nada satis- } \\
\text { fecho }\end{array}$ & 0,042 & 0,487 & 0,628 & 0,005 & 0,039 & 0,124 & 0,519 & 0,449 & 0,968 \\
\hline Total activo & 1,000 & & & 0,084 & 1,000 & 1,000 & & & \\
\hline \multicolumn{10}{|c|}{ Puntos de columnab } \\
\hline \multirow{3}{*}{ Salud } & \multirow{3}{*}{ Masa } & \multicolumn{2}{|c|}{$\begin{array}{l}\text { Puntuación en } \\
\text { dimensión }\end{array}$} & \multirow{3}{*}{ Inercia } & \multicolumn{5}{|c|}{ Contribución } \\
\hline & & 1 & 2 & & \multicolumn{2}{|c|}{$\begin{array}{l}\text { Del punto en la inercia de } \\
\text { dimensión }\end{array}$} & \multicolumn{3}{|c|}{ De la dimensión en la inercia del punto } \\
\hline & & & & & 1 & 2 & 1 & 2 & Total \\
\hline Muy buena & 0,232 & $-0,854$ & 0,210 & 0,045 & 0,661 & 0,077 & 0,969 & 0,031 & 1,000 \\
\hline Buena & 0,552 & 0,127 & $-0,284$ & 0,008 & 0,035 & 0,333 & 0,279 & 0,720 & 0,999 \\
\hline Aceptable & 0,188 & 0,590 & 0,387 & 0,021 & 0,255 & 0,210 & 0,817 & 0,182 & 0,999 \\
\hline Mala & 0,025 & 0,715 & 1,127 & 0,007 & 0,049 & 0,236 & 0,436 & 0,563 & 1,000 \\
\hline Muy mala & 0,003 & $-0,150$ & 2,620 & 0,003 & 0,000 & 0,144 & 0,006 & 0,933 & 0,939 \\
\hline Total activo & 1,000 & & & 0,084 & 1,000 & 1,000 & & & \\
\hline
\end{tabular}

a. 12 grados de libertad

b. Normalización Simétrica

Fuente: Elaboración propia a partir de la 6ạ Encuesta Europea de Condiciones de Trabajo-España 2015. 
TABLA 5

REGRESIÓN LOGÍSTICA BINARIA.

ESTIMACIONES DE LOS PARÁMETROS.

VARIABLE DEPENDIENTE: SATISFACCIÓN CON EL TRABAJO

\begin{tabular}{|c|c|c|c|c|c|c|c|}
\hline \multirow{2}{*}{\multicolumn{2}{|c|}{ Satisfecho-muy satisfecho ${ }^{a}$}} & \multirow{3}{*}{$\begin{array}{l}\text { B } \\
-7,620\end{array}$} & \multirow{3}{*}{$\begin{array}{l}\text { Error estándar } \\
\text { 0,040 }\end{array}$} & \multirow{3}{*}{$\begin{array}{r}\text { Sig } \\
0,000\end{array}$} & \multirow{3}{*}{$\operatorname{Exp}(B)$} & \multicolumn{2}{|c|}{ I. Confianza 95\% } \\
\hline & & & & & & \multirow{2}{*}{ Límite inferior } & \multirow{2}{*}{ Límite superior } \\
\hline & Intersección & & & & & & \\
\hline Edad & $\begin{array}{r}\text { Edad en } 3 \\
\text { rangos }\end{array}$ & 0,384 & 0,001 & 0,000 & 1,468 & 1,464 & 1,472 \\
\hline \multirow{2}{*}{ Género } & Hombre & 0,044 & 0,002 & 0,000 & 1,045 & 1,041 & 1,049 \\
\hline & Mujer & $0^{b}$ & . & & . & . & . \\
\hline \multirow{5}{*}{$\begin{array}{l}\text { Creo que mi } \\
\text { sueldo es } \\
\text { apropiado }\end{array}$} & $\begin{array}{r}\text { Totalmente de } \\
\text { acuerdo }\end{array}$ & 1,975 & 0,005 & 0,000 & 7,208 & 7,145 & 7,272 \\
\hline & $\begin{array}{r}\text { Parcialmente } \\
\text { de acuerdo }\end{array}$ & 1,525 & 0,003 & 0,000 & 4,594 & 4,566 & 4,623 \\
\hline & $\begin{array}{r}\mathrm{Ni} \text { acuerdo ni } \\
\text { desacuerdo }\end{array}$ & 1,157 & 0,003 & 0,000 & 3,180 & 3,161 & 3,199 \\
\hline & $\begin{array}{r}\text { Parcialmente } \\
\text { desacuerdo }\end{array}$ & 0,557 & 0,003 & 0,000 & 1,746 & 1,737 & 1,754 \\
\hline & $\begin{array}{r}\text { Totalmente en } \\
\text { desacuerdo }\end{array}$ & $0^{b}$ & . & . & . & . & . \\
\hline \multirow{5}{*}{$\begin{array}{l}\text { Mi empleo me } \\
\text { ofrece buenas } \\
\text { posibilidades } \\
\text { de ascenso } \\
\text { profesional }\end{array}$} & $\begin{array}{r}\text { Totalmente de } \\
\text { acuerdo }\end{array}$ & 0,343 & 0,004 & 0,000 & 1,410 & 1,398 & 1,421 \\
\hline & $\begin{array}{r}\text { Parcialmente } \\
\text { de acuerdo }\end{array}$ & 1,061 & 0,003 & 0,000 & 2,890 & 2,871 & 2,909 \\
\hline & $\begin{array}{r}\mathrm{Ni} \text { acuerdo ni } \\
\text { desacuerdo }\end{array}$ & 0,355 & 0,003 & 0,000 & 1,427 & 1,419 & 1,435 \\
\hline & $\begin{array}{r}\text { Parcialmente } \\
\text { desacuerdo }\end{array}$ & 0,019 & 0,003 & 0,000 & 1,019 & 1,013 & 1,024 \\
\hline & $\begin{array}{r}\text { Totalmente en } \\
\text { desacuerdo }\end{array}$ & $0^{b}$ & . & . & . & . & . \\
\hline \multirow{5}{*}{$\begin{array}{l}\text { Recibo el } \\
\text { reconoci- } \\
\text { miento que } \\
\text { merezco por } \\
\text { mi trabajo }\end{array}$} & $\begin{array}{r}\text { Totalmente de } \\
\text { acuerdo }\end{array}$ & 1,624 & 0,004 & 0,000 & 5,073 & 5,029 & 5,118 \\
\hline & $\begin{array}{r}\text { Parcialmente } \\
\text { de acuerdo }\end{array}$ & 0,914 & 0,003 & 0,000 & 2,495 & 2,479 & 2,512 \\
\hline & $\begin{array}{r}\text { Ni acuerdo ni } \\
\text { desacuerdo }\end{array}$ & 0,402 & 0,003 & 0,000 & 1,494 & 1,485 & 1,504 \\
\hline & $\begin{array}{r}\text { Parcialmente } \\
\text { desacuerdo }\end{array}$ & $-0,213$ & 0,003 & 0,000 & 0,808 & 0,803 & 0,814 \\
\hline & $\begin{array}{l}\text { Totalmente en } \\
\text { desacuerdo }\end{array}$ & $0^{b}$ & . & . & . & . & . \\
\hline
\end{tabular}




\begin{tabular}{|c|c|c|c|c|c|c|c|}
\hline \multirow{5}{*}{$\begin{array}{l}\text { En general me } \\
\text { llevo bien con } \\
\text { mis compañe- } \\
\text { ros de trabajo }\end{array}$} & $\begin{array}{r}\text { Totalmente de } \\
\text { acuerdo }\end{array}$ & 1,599 & 0,008 & 0,000 & 4,946 & 4,870 & 5,023 \\
\hline & $\begin{array}{r}\text { Parcialmente } \\
\text { de acuerdo }\end{array}$ & 1,001 & 0,008 & 0,000 & 2,722 & 2,679 & 2,766 \\
\hline & $\begin{array}{l}\mathrm{Ni} \text { acuerdo ni } \\
\text { desacuerdo }\end{array}$ & 0,568 & 0,008 & 0,000 & 1,765 & 1,736 & 1,795 \\
\hline & $\begin{array}{l}\text { Parcialmente } \\
\text { desacuerdo }\end{array}$ & 0,760 & 0,010 & 0,000 & 2,138 & 2,098 & 2,179 \\
\hline & $\begin{array}{l}\text { Totalmente en } \\
\text { desacuerdo }\end{array}$ & $0^{\mathrm{b}}$ & . & . & . & . & \\
\hline \multirow{5}{*}{$\begin{array}{l}\text { La organiza- } \\
\text { ción en la que } \\
\text { trabajo me } \\
\text { motiva para } \\
\text { dar lo mejor } \\
\text { de mí en el } \\
\text { trabajo }\end{array}$} & $\begin{array}{c}\text { Totalmente de } \\
\text { acuerdo }\end{array}$ & 1,264 & 0,004 & 0,000 & 3,541 & 3,512 & 3,569 \\
\hline & $\begin{array}{l}\text { Parcialmente } \\
\text { de acuerdo }\end{array}$ & 1,235 & 0,003 & 0,000 & 3,438 & 3,416 & 3,460 \\
\hline & $\begin{array}{l}\text { Ni acuerdo ni } \\
\text { desacuerdo }\end{array}$ & 0,894 & 0,003 & 0,000 & 2,446 & 2,431 & 2,461 \\
\hline & $\begin{array}{l}\text { Parcialmente } \\
\text { desacuerdo }\end{array}$ & 0,424 & 0,003 & 0,000 & 1,528 & 1,518 & 1,538 \\
\hline & $\begin{array}{c}\text { Totalmente en } \\
\text { desacuerdo }\end{array}$ & $0^{\mathrm{b}}$ & . & . & . & . & \\
\hline \multirow{5}{*}{$\begin{array}{c}\text { ¿Cómo es } \\
\text { su salud en } \\
\text { general? Diría } \\
\text { que es }\end{array}$} & Muy buena & 1,845 & 0,035 & 0,000 & 6,325 & 5,901 & 6,780 \\
\hline & Buena & 1,462 & 0,035 & 0,000 & 4,316 & 4,027 & 4,625 \\
\hline & Aceptable & 1,388 & 0,035 & 0,000 & 4,007 & 3,739 & 4,295 \\
\hline & Mala & 0,522 & 0,036 & 0,000 & 1,685 & 1,571 & 1,808 \\
\hline & Muy mala & $0^{\mathrm{b}}$ & . & . & . & . & . \\
\hline \multirow{2}{*}{$\begin{array}{l}\text { Usted trabaja } \\
\text { por turnos }\end{array}$} & Sí & $-0,046$ & 0,002 & 0,000 & 0,955 & 0,952 & 0,959 \\
\hline & No & $0^{b}$ & . & & & & \\
\hline \multirow{3}{*}{$\begin{array}{l}\text { Tipo de } \\
\text { contrato }\end{array}$} & Indefinido & 1,510 & 0,005 & 0,000 & 4,525 & 4,478 & 4,572 \\
\hline & Temporal & 1,008 & 0,005 & 0,000 & 2,740 & 2,712 & 2,769 \\
\hline & Sin contrato & $0^{\mathrm{b}}$ & . & & & & \\
\hline \multirow{4}{*}{$\begin{array}{l}\text { Sector de } \\
\text { actividad }\end{array}$} & Agrario & $-0,185$ & 0,005 & 0,000 & 0,831 & 0,823 & 0,840 \\
\hline & Industria & 0,067 & 0,003 & 0,000 & 1,069 & 1,064 & 1,075 \\
\hline & Construcción & $-0,550$ & 0,004 & 0,000 & 0,577 & 0,573 & 0,582 \\
\hline & Servicios & $0^{\mathrm{b}}$ & & . & . & . & . \\
\hline \multirow{2}{*}{$\begin{array}{l}\text { ¿Trabaja como } \\
\text { empleado por } \\
\text { cuenta ajena o } \\
\text { es autónomo? }\end{array}$} & Empleado & 1,827 & 0,017 & 0,000 & 6,217 & 6,017 & 6,423 \\
\hline & Autónomo & $0^{\mathrm{b}}$ & . & & & & \\
\hline
\end{tabular}

a. La categoría de referencia es Nada satisfecho-no muy satisfecho.

b. Este parámetro está establecido en 0 porque es redundante.

Fuente: Elaboración propia a partir de la 6a Encuesta Europea de Condiciones de Trabajo-España 2015. 
Hipótesis 4: Efecto del tiempo en las relaciones entre las variables trabajo a turnos, salud y satisfacción laboral.

Este estudio es de tipo transversal, pero creemos que si se hiciese el seguimiento de un mismo grupo de trabajadores a lo largo del tiempo, los efectos serían más notorios. Así lo demuestra el estudio realizado por Koller et alii (1990). Estos autores argumentan que los problemas causados por el trabajo a turnos podrían aumentar con la duración de la exposición. Para estudiar esta cuestión, llevaron a cabo un estudio longitudinal de cinco años en un colectivo de trabajadores. La calidad del sueño después de un horario nocturno se consideró significativamente peor para los trabajadores a turnos. En este contexto, hubo un aumento en la sensación de fatiga entre estos trabajadores. La satisfacción de los trabajadores a turnos en su trabajo y especialmente en sus vidas fue disminuyendo a lo largo de los cinco años, mientras que tendió a aumentar entre los trabajadores diurnos. Se estableció un indicador de salud a partir de la sintomatología recogida a través de entrevistas médicas. La comparación en los cinco años mostró un deterioro significativo en el estado de salud de los trabajadores a turnos. Por todo ello, el trabajo a turnos aparece como un factor desestabilizador, produciendo a la larga, efectos negativos que pueden tener implicaciones sobre el bienestar y la salud.

\section{Discusión}

En el epígrafe de resultados (desarrollo de la hipótesis 1) se vio que los trabajadores a turnos rotativos consideran que su salud no es tan buena comparada con el colectivo total $(-1,2 \%)$, aunque nuestras expectativas iniciales nos hacían suponer que esta cifra iba a ser más pequeña. Conviene precisar que la salud es la declarada en el momento de la entrevista y aunque los riesgos existen, los efectos de trabajar a turnos sobre la salud no suelen producirse de manera inmediata. Lo anterior nos permite deducir, por tanto, que los turnos rotativos y nocturnos son los que implican mayor riesgo para la salud. Por ello, resulta necesaria una coordinación efectiva entre diferentes administraciones, especialmente la sanitaria y la del trabajo (Benavides et alii, 2017) para abordar esta problemática. Se trata de una perspectiva desde la cual se pueda hilvanar una relación entre la persona que trabaja, su salud y el ámbito en el cual realiza su tarea (Martin \& Spinelli, 2011).

En cuanto a la hipótesis 2, los trabajadores a turnos rotativos se encuentran menos satisfechos $(-4,7 \%)$ que el conjunto de los trabajadores; esta teoría está en la línea de Juárez-Adauta (2012), en cuyo estudio, la satisfacción laboral fue mayor en los trabajadores de jornada continua (acumulada), seguidos por los trabajadores de 
turnos matutinos y vespertinos, siendo los trabajadores del turno nocturno los que presentaron menor satisfacción laboral $(p<0,002)$.

A la vista de los resultados obtenidos en el desarrollo de las hipótesis 1 y 2 se constata que en los trabajadores a turnos rotativos el efecto negativo es más acusado en la satisfacción que en la salud.

Para argumentar la hipótesis 3, mediante el análisis de correspondencias-metodología utilizada en satisfacción laboral por algunos autores (Sánchez-Sellero et alii, 2018; Parada et alii, 2005), se comprobó que las variables salud y satisfacción laboral no son independientes, existiendo una relación significativa entre ellas (véase $\mathrm{p}=0,000$ en Tabla 4); este resultado es coherente con lo visto en la Tabla 3. De igual modo, otros autores como Sánchez et alii (2017) utilizan este procedimiento en el estudio de los riesgos laborales en las empresas.

En la Tabla 4, la inercia total representa la explicación total de las dimensiones o ejes (explican un total de 0,084). Aunque el valor de la inercia (cada inercia=valor propio al cuadrado) es pequeño, entre las tres dimensiones explican el $100 \%$ de la varianza. El número de dimensiones es una unidad inferior al número de categorías de la variable con menos categorías; dado que la variable satisfacción tiene 4 categorías y salud tiene 5 , esto hace que las dimensiones sean 3 . Se observa que solo la primera dimensión contribuye un $78,5 \%$ a dicha inercia y que las dos primeras contribuyen un $99,8 \%$, por lo que las dependencias observadas en la tabla de contingencia están reflejadas adecuadamente por esas dimensiones.

El análisis de las contribuciones absolutas y relativas (últimas 5 columnas de los puntos de fila y puntos de columna, Tabla 4) informa cuáles son las categorías más relacionadas con cada dimensión, mientras que el signo de las coordenadas (columnas 3 y 4) sitúan cada categoría en el plano; por ejemplo, la categoría "muy buena salud" se representa en el plano (Figura 2) en el punto $(-0,854,0,210)$. En la última columna de las contribuciones relativas, los valores iguales a 1 o cercanos a la unidad, reflejan la alta calidad de todos los puntos sobre las 2 dimensiones.

En la Figura 2 se demostraron asociaciones entre las categorías "buena salud" y considerarse "satisfecho" en el trabajo; de igual modo, los que gozan de "muy buena salud" son los que se encuentran "muy satisfechos" laboralmente. Repetido este análisis para cada uno de los turnos de trabajo, se constató un resultado similar, así como para el total de los trabajadores. En esta línea se encuentran los resultados de Martín et alii (2007); éstos muestran que los empleados que se sienten estresados, 
fatigados, y poco satisfechos perciben su entorno laboral de forma más adversa, y de modo similar se obtuvieron para aquellos trabajadores que gozan de poca salud.

En la Tabla 5 se observa el signo negativo del parámetro "Usted trabaja por turnos: sí, ilustrativo de que en los trabajadores a turnos disminuye la satisfacción; además, $\operatorname{Exp}(B)$ conocido como odds-ratio indica que la satisfacción de estos trabajadores es $1 / 0,955=1,047$ veces menor que aquellos que no están a turnos. Los trabajadores que declaran gozar de muy buena salud están 6,325 veces más satisfechos en el trabajo que la categoría de referencia (salud muy mala). Este modelo presenta los tests de bondad de ajuste siguientes: Pseudo $R^{2}$ de Cox \& Snell igual a 0,282, de Nagelkerke de 0,465 y de McFadden de 0,355, valores que consideramos adecuados, y que reflejan una correcta capacidad explicativa del modelo. El porcentaje de clasificación del modelo es del 87,4\%, considerándolo satisfactorio.

Con respecto a la hipótesis 4 es importante tener en cuenta que muchas relaciones causales "necesitan tiempo" para ser probadas porque, entre otras razones, la influencia de una variable sobre otra no ocurre solo una vez (Vázquez, 2018). En este caso, consideramos útil el uso de datos de panel, donde partiendo del mismo colectivo de trabajadores se pudiese comprobar en distintos años qué puntuaciones dan a su satisfacción en el trabajo así como a la valoración de su salud. No disponemos de información de datos públicos para este objetivo ya que las muestras de la Encuesta Europea de Condiciones de Trabajo en otros años no corresponden a los mismos trabajadores; necesitaríamos crear una misma muestra de trabajadores a los que se les hiciese el seguimiento para tal finalidad.

\section{Conclusiones}

En este artículo realizamos un análisis estadístico con datos de EWCS, España, partiendo del ámbito descriptivo, y llegando al recurso de la regresión estadística tipo Logit, aplicable al objeto que trata y la relación que se quiere demostrar. Esta metodología proporciona un tratamiento estadístico más riguroso de las hipótesis formuladas.

Hemos comprobado que el trabajo a turnos conlleva no solo porcentajes menores de satisfacción laboral, sino que también se asocia a porcentajes inferiores en la percepción de salud. El colectivo más perjudicado por los efectos negativos del trabajo a turnos es el de los trabajadores a turnos rotativos, siendo el efecto más acusado en la satisfacción que en la salud. De todos modos, nuestras expectativas iniciales presuponían unas cifras marcadamente más bajas con respecto a los traba- 
jadores que realizan un trabajo convencional. Esta reflexión nos conduce a pensar que el tiempo es un elemento relevante y a tener en consideración en este estudio triangular (trabajo a turnos, salud y satisfacción).

En este trabajo intentamos relacionar tres conceptos: turnos de trabajo, salud y satisfacción laboral, encontrando que aunque las tres correlaciones posibles son significativas, la correlación mayor es la existente entre salud y satisfacción laboral, dos percepciones de tipo subjetivo que se asocian de una manera directa.

Mediante la regresión logística interpretamos que la satisfacción de los trabajadores a turnos es 1,047 veces menor que los que no están a turnos, mientras que los que gozan de muy buena salud están 6,325 veces más satisfechos en el trabajo que la categoría de referencia (personas que creen tener muy mala salud).

La literatura demuestra empíricamente que determinadas condiciones laborales puedan afectar a la salud, como se ha visto en este trabajo; pero más concretamente, se ha evidenciado una asociación entre sentirse sano y sentirse satisfecho. Este conocimiento tiene implicaciones prácticas ya que al existir una correlación elevada entre salud y satisfacción laboral, una mejora en los factores determinantes de la satisfacción (vinculados a características propias del trabajo), podrían tener una repercusión positiva sobre la salud de los trabajadores. Aunque también se podría formular ese planteamiento al revés, es decir, ¿la salud puede condicionar la satisfacción laboral? O dicho de otro modo, ¿mejoras en la percepción del estado de salud conduciría a un aumento en la satisfacción en el trabajo? La interrelación se ha comprobado, un paso posterior trataría de ahondar en el origen y fundamentación de estas asociaciones.

La limitación de este trabajo es que se trata de un análisis de tipo transversal, sería necesario un estudio de datos de panel para ampliar nuestras conclusiones. Aunque no tenemos información disponible para realizar un análisis longitudinal de este tipo, sí hemos mostrado literatura que contesta a nuestra hipótesis de trabajo y según la cual la permanencia en el trabajo a turnos hace incrementar los efectos negativos sobre la salud y sobre la satisfacción de los trabajadores.

Como ya apuntamos en el marco teórico, pudimos comprobar que el trabajo no es neutral respecto a la salud, y por ello resulta necesaria una coordinación efectiva entre las administraciones sanitarias y del trabajo. Se trata de una perspectiva desde la cual pueda desarrollarse una relación entre el trabajador, su salud y el ámbito laboral en el cual realiza su tarea. Por consiguiente, distintos temas relativos al 
mundo laboral (trabajo a turnos, prevención y seguridad en el trabajo etc.) tienen su repercusión en la salud de los trabajadores, y por tanto, existen vinculaciones entre el ámbito sanitario y laboral.

\section{Referencias}

BENAVIDES, F. G.; DELCLÓS, J.; SERRA, C. Estado de bienestar y salud pública: el papel de la salud laboral. Gaceta Sanitaria, v. 32, n. 4, p. 377-380, 2017.

BERNSTORFF, V. Relações entre satisfação, competência, saúde e absenteísmo no trabalho em uma grande instituição bancária pública. Sociedade e Estado, v. 23, n. 1, p. 189-190, 2008.

BOLETIN OFICIAL DEL ESTADO - BOE. Disposición 11430 del BOE n. 255 de 2015. Real Decreto Legislativo 2/2015, de 23 de octubre, por el que se aprueba el texto refundido de la ley del Estatuto de los Trabajadores (24 Oct. 2015).

BORIA-REVERTER, S.; CRESPI-VALLBONA, M.; MASCARILLA-MIRÓ, O. Variables determinantes de la satisfacción laboral en España. Cuadernos de Economía, v. 35, n. 97, p. 9-16, 2012.

BRASIL, CONSOLIDAÇÃO DAS LEIS DO TRABALHO. Decreto-Lei n. 5.452, de 1 으 de maio de 1943.

BRASIL, CONSTITUIÇÃO DA REPÚBLICA FEDERATIVA DO BRASIL de 1988. Texto constitucional promulgado em 5 de outubro de 1988, atualizado até a Emenda Constitucional n. 101, de 03 de julho de 2019. São Paulo, Set. 2019

DALL'ORA, C.; BALL, J.; RECIO-SAUCEDO, A.; GRIFFITHS, P. Characteristics of shift work and their impact on employee performance and wellbeing: a literature review. International Journal of Nursing Studies, n. 57, p. 12-27, 2016.

DUEÑAS, D.; IGLESIAS, C.; LLORENTE, R. Job quality, job satisfaction and services in Spain. Journal of Innovation Economics, v. 1, n. 5, p. 145-166, 2010.

ENCUESTA EUROPEA DE CONDICIONES DE TRABAJO, 6a ECWS - España 2015. Madrid: Instituto Nacional de Seguridad e Higiene en el Trabajo (INSHT), 2017.

FERNÁNDEZ, J.; PIÑOL, E. Horario laboral y salud: consecuencias psicológicas de los turnos de trabajo. Revista de psicopatología y psicología clínica, v. 5, n. 3, p. 207222, 2000. 
FERRI, P.; GUADI, M.; MARCHESELLI, L.; BALDUZZI, S.; MAGNANI, D.; DI LORENZO, $R$. The impact of shift work on the psychological and physical health of nurses in a general hospital: a comparison between rotating night shifts and day shifts. Risk Management and Healthcare Policy, v. 14, n. 9, p. 203-211, 2016.

GÁlLEGO, J.; TOLEDO, J. B.; URRESTARAZU, E.; IRIARTE, J. Clasificación de los trastornos del sueño. Anales del Sistema Sanitario de Navarra, v. 30 (Supl. 1), p. 19-36, 2007.

GARCÍA, A. M.; BOIX, P.; BENAVIDES, F. G.; GADEA, R.; RODRIGO, F.; SERRA, C. Participación para mejorar las condiciones de trabajo: evidencias y experiencias. Gaceta Sanitaria, v. 30 (Supl. 1), p. 87-92, 2016.

GIL-MONTE, P. R. Riesgos psicosociales en el trabajo y salud ocupacional. Revista Peruana de Medicina Experimental y Salud Pública, v. 29, n. 2, p. 237-241, 2012.

HITKA, M.; ZÁVADSKÁ, Z.; JELAČIĆ, D.; BALÁŽOVÁ, Ž. Qualitative indicators of company employee satisfaction and their development in a particular period of time. Drvna Industrija, v. 66, n. 3, p. 235-239, 2015.

IGLESIAS, C.; LLORENTE, R.; DUEÑAS, D. Calidad del empleo y satisfacción laboral en las regiones españolas. Un estudio con especial referencia a la Comunidad de Madrid. Investigaciones Regionales, v. 19, p. 25-49, 2011.

ISKRA-GOLEC, I.; SMITH, L.; WILCZEK-RUŻYCZKA, E.; SIEMIGINOWSKA, P.; WĄTROBA, $J$. Shift schedule, work-family relationships, marital communication, job satisfaction and health among transport service shift workers. International Journal of Occupational Medicine and Environmental Health, v. 30, n. 1, p. 121-131, 2017.

JUÁREZ-ADAUTA, S. Clima organizacional y satisfacción laboral. Revista Médica del Instituto Mexicano del Seguro Social, v. 50, n. 3, p. 307-314, 2012.

KHAMMAR, A.; AMJAD, R. N.; ROHANI, M.; YARI, A.; NOROOZI, M.; POURSADEGHIAN, A.; HAMI, M.; POURSADEGHIYAN, M. Survey of shift work disorders and occupational stress among nurses: A cross-sectional study. Annals of Tropical Medicine and Public Health, v. 10, n. 4, p. 978-984, 2017.

KOLLER, M.; KUNDI, M.; HAIDER, M.; CERVINKA, R.; FRIZA, H. Changements survenus en cinq ans dans la vie familiale, la satisfaction et la santé de travailleurs postés. Le Travail Humain, v. 53, n. 2, p. 153-174, 1990.

LORINCOVÁ, S.; ŠTARCHOŇ, P.; WEBEROVÁ, D.; HITKA, M.; LIPOLDOVÁ, M. Employee Motivation as a Tool to Achieve Sustainability of Business Processes. Sustainability, v. 11, n. 13, p. 3509, 2019. 
MARTIN, A. L.; SPINELLI, H. Para que el hombre vuelva a cantar mientras trabaja. EI Instituto de Medicina del Trabajo (IMT) y la salud de los trabajadores. Salud Colectiva, v. 7, n. 2, p. 177-197, 2011.

MARTÍN, J.; LUCEÑO, L.; JAÉN, M.; RUBIO, S. Relación entre factores psicosociales adversos, evaluados a través del cuestionario multidimensional Decore, y salud laboral deficiente. Psicothema, v. 19, n. 1, p. 95-101, 2007.

PARADA, M. E.; MORENO, R.; MEJÍAS, Z.; RIVAS, A.; RIVAS, F.; CERRADA, J.; RIVAS, F. Satisfacción laboral y síndrome de Burnout en el personal de enfermería del Instituto Autónomo Hospital Universitario Los Andes (lahula), Mérida, Venezuela, 2005. Revista Facultad Nacional de Salud Pública, v. 23, n. 1, p. 33-45, 2005.

PARRA, M. Conceptos básicos en salud laboral. Santiago de Chile: OIT, 2003.

PERAITA-ADRADOS, R. Avances en el estudio de los trastornos del sueño. Revista Neurológica, v. 40, n. 8, p. 485-491, 2005.

PORCEL-GÁlVEZ, A. M.; MARTÍNEZ-LARA, C.; GIL-GARCÍA, E.; GRAO-CRUCES A. Construcción y validación del cuestionario G_Clinic para medir la satisfacción laboral en profesionales de enfermería de las unidades de gestión clínica. Revista Española de Salud Pública, v. 88, n. 3, p. 419-428, 2014.

ROBLES-GARCÍA, M.; DIERSSEN-SOTOS, T.; MARTÍNEZ-OCHOA, E.; HERRERA-CARRAL, P.; DÍAZ-MENDI, A. R.; LLORCA-DÍAZ, J. Variables relacionadas con la satisfacción laboral: Un estudio transversal a partir del modelo EFQM. Gaceta Sanitaria, v. 19, n. 2, p. 127-134, 2005.

RODRÍGUEZ, C. A. La salud de los trabajadores: entre la ciencia y la ética - Editorial. Salud Colectiva, v. 9, n. 2, p. 133-137, 2013.

SÁNCHEZ, A. M.; SÁNCHEZ, F. J.; RUIZ-MUÑOZ, D. Riesgos laborales en las empresas de residuos sólidos en Andalucía: una perspectiva de género. Saúde e Sociedade, v. 26, n. 3, p. 798-810, 2017.

SÁNCHEZ-SELLERO, M. C.; SÁNCHEZ-SELLERO, P.; CRUZ-GONZÁLEZ, M. M.; SÁNCHEZ-SELLERO, F. J. Características organizacionales de la satisfacción laboral en España. Revista de Administração de Empresas (RAE), v. 54, n. 5, p. 537-547, 2014.

SÁNCHEZ-SELLERO, M. C.; SÁNCHEZ-SELLERO, P.; CRUZ-GONZÁLEZ, M. M.; SÁNCHEZ-SELLERO, F. J. Relación entre sobrecualificación y satisfacción laboral durante la crisis española de 2008. Contaduría y Administración, v. 63, n. 2, p. 1-20, 2018. 
SANTOS, F.; GUILLÉN, C.; MONTALBÁN, F. M. Contrato de trabalho, compromisso e satisfação: moderação pela empregabilidade. Revista de Administração de Empresas (RAE), v. 52, n. 3, p. 345-359, 2012.

ŠIMUNIĆ, A.; GREGOV, L. Conflict between work and family roles and satisfaction among nurses in different shift systems in Croatia: a questionnaire survey. Archives of Industrial Hygiene and Toxicology, v. 63, n. 2, p. 189-197, 2012.

VÁZQUEZ, X. H. The poetry of intriguing ideas, logical arguments and rigorous methods in management. Business Research Quarterly (BRQ), v. 21, n. 1, p. 1-10, 2018. 
